\title{
Correction to: Expression of the immune checkpoint VISTA in breast cancer
}

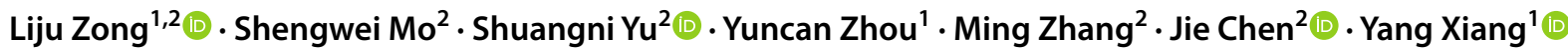

Published online: 18 June 2020

(c) Springer-Verlag GmbH Germany, part of Springer Nature 2020

\section{Correction to: Cancer Immunology, Immunotherapy https://doi.org/10.1007/s00262-020-02554-3}

The original version of this article unfortunately contained a mistake. The correct information is given below.

In abstract , the second and third sentences should read as

This study aimed to investigate the expression of VISTA and its association with clinicopathologic parameters as well as with the key immune markers including programmed cell death-1 (PD-1) and PD-1 ligand-1 (PD-L1) in invasive ductal carcinoma (IDC) of the breast.

Immunohistochemistry was used to detect VISTA, PD-1, PD-L1, and CD8 in tissue microarrays from 919 patients with IDC $(N=341$ in the exploratory cohort and $N=578$ in the validation cohort).

In section "Evaluation of immunostaining", the fifth sentence of the second paragraph should read as

The original article can be found online at https://doi.org/10.1007/ s00262-020-02554-3.

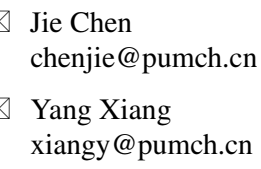

1 Department of Obstetrics and Gynecology, Peking Union Medical College Hospital, Chinese Academy of Medical Sciences and Peking Union Medical College, No.1 Shuaifuyuan, Dongcheng District, Beijing 100730, China

2 Department of Pathology, Peking Union Medical College Hospital, Chinese Academy of Medical Sciences and Peking Union Medical College, No.1 Shuaifuyuan, Dongcheng District, Beijing 100730, China
Luminal A was defined as ER $+(\geq 1 \%)$ or $\mathrm{PR}+(\geq 1 \%)$, HER2-, and low Ki67( $<14 \%)$; luminal B was defined as $\mathrm{ER}+($ or PR +) and HER2 + or high Ki67 ( $\geq 14 \%)$; HER2enriched (HER2E) was defined as HER + , ER-, and PR-; and basal-like was defined as ER-, PR-, and HER2- with either EGFR + or CK5/6 + .

Publisher's Note Springer Nature remains neutral with regard to jurisdictional claims in published maps and institutional affiliations. 